\title{
Wire-grid polarizer using galvanic growth technology: demonstration of a wide spectral and angular bandwidth component with high extinction ratio (Erratum)
}

Isabelle Verrier, ${ }^{\mathrm{a}}$ Thomas Kämpfe, ${ }^{\mathrm{a}}$ Frederic Celle, ${ }^{\mathrm{a}}$ Anthony Cazier, ${ }^{\mathrm{a}}$ Koceilla Yadel, ${ }^{\mathrm{a}}$ Markus Guttmann, ${ }^{\mathrm{b}}$ Barbara Matthis, ${ }^{\mathrm{b}}$ Janne Laukkanen, ${ }^{\mathrm{c}}$ Frédéric Lacour, ${ }^{\mathrm{d}}$ Colette Veillas, ${ }^{\mathrm{a}}$ Stéphanie Reynaud, ${ }^{\mathrm{a}}$ Olivier Parriaux, ${ }^{\mathrm{a}}$ and Yves Jourlin ${ }^{\mathrm{a}, *}$

${ }^{a}$ Université de Lyon, Laboratoire Hubert Curien UMR CNRS 5516, Saint-Etienne, France

${ }^{\mathrm{b}}$ Karlsruhe Institute of Technology, Institute of Microstructure Technology Hermann-von-Helmholtz-Platz 1, Eggenstein-Leopoldshafen, Germany

${ }^{c}$ University of Eastern Finland, Department of Physics and Mathematics, Joensuu, Finland

${ }^{\mathrm{d}}$ Exxelia Group, Eurofarad Department Capteurs et Systèmes Associés, Chanteloup-en-Brie, France

[DOI: 10.1117/1.OE.59.12.129801]

Originally published on 28 April 2015, this article [Opt. Eng. 54(4), 047105 (2015) DOI: 10 .1117/1.OE.54.4.047105] left off an author. Koceilla Yadel performed optical measurements on the polarizer, which was reported in an SPIE conference [Proc. SPIE 9130, 913008 (2014) DOI: 10.1117/12.2051429]. For the conference paper, he was listed as a co-author. These results were subsequently published in the Optical Engineering paper, so he has been added as an author.

The paper was corrected online on 4 December 2020.

*Address all correspondence to: Yves Jourlin, E-mail: yves.jourlin@univ-stetienne.fr 